\title{
Centralizers of coprime automorphisms of finite groups
}

\author{
Cristina Acciarri · Pavel Shumyatsky
}

Received: 1 December 2011 / Accepted: 12 May 2012 / Published online: 26 May 2012

(C) Fondazione Annali di Matematica Pura ed Applicata and Springer-Verlag 2012

\begin{abstract}
Let $A$ be an elementary abelian group of order $p^{k}$ with $k \geq 3$ acting on a finite $p^{\prime}$-group $G$. The following results are proved. If $\gamma_{k-2}\left(C_{G}(a)\right)$ is nilpotent of class at most $c$ for any $a \in A^{\#}$, then $\gamma_{k-2}(G)$ is nilpotent and has $\{c, k, p\}$-bounded nilpotency class. If, for some integer $d$ such that $2^{d}+2 \leq k$, the $d$ th derived group of $C_{G}(a)$ is nilpotent of class at most $c$ for any $a \in A^{\#}$, then the $d$ th derived group $G^{(d)}$ is nilpotent and has $\{c, k, p\}$-bounded nilpotency class.
\end{abstract}

Keywords Finite groups · Associated Lie algebras · Automorphisms · Centralizers

Mathematics Subject Classification Primary 20D45; Secondary 20F40

\section{Introduction}

Let $G$ be a group admitting an action by a group $A$. We denote by $C_{G}(A)$ the set $\{x \in G$ | $x^{a}=x$ for any $\left.a \in A\right\}$, the centralizer of $A$ in $G$. In this paper we deal with the case where $A$ is a noncyclic elementary abelian $p$-group and $G$ is a finite $p^{\prime}$-group. Let $A^{\#}$ denote the set of non-identity elements of $A$. It follows from the classification of finite simple groups that if $C_{G}(a)$ is soluble for any $a \in A^{\#}$, then so is the group $G$ (see [4]). The case $|A| \geq p^{3}$ does not require the classification: the result follows from Glauberman's theorem on soluble signalizer functors [2]. In certain specific situations much more can be said about the structure of $G$.

This research was supported by CNPq-Brazil.

C. Acciarri · P. Shumyatsky $(\varangle)$

Department of Mathematics, University of Brasilia,

Brasilia, DF 70910-900, Brazil

e-mail: pavel@unb.br

C. Acciarri

e-mail: acciarricristina@yahoo.it 
Ward showed that if $A$ has rank at least 3, and if $C_{G}(a)$ is nilpotent for any $a \in A^{\#}$, then the group $G$ is nilpotent [7]. Another of Ward's results is that if $A$ has rank at least 4 , and if $C_{G}(a)^{\prime}$ is nilpotent for any $a \in A^{\#}$, then the derived group $G^{\prime}$ is nilpotent [8].

Later the second author of the present paper found that if under these assumptions $C_{G}(a)$ is nilpotent of class at most $c$ (respectively $C_{G}(a)^{\prime}$ is nilpotent of class at most $c$ ) for any $a \in A^{\#}$, then the nilpotency class of $G$ (respectively of $G^{\prime}$ ) is $\{c, p\}$-bounded [6]. Throughout the article we use the term " $\{a, b, c, \ldots\}$-bounded" to mean "bounded from above by some function depending only on the parameters $a, b, c, \ldots$."

Let us denote by $\gamma_{i}(H)$ the $i$ th term of the lower central series of a group $H$ and by $H^{(i)}$ the $i$ th term of the derived series of $H$. In [6] it was conjectured that the above results should be a part of a more general phenomenon.

Conjecture 11 Let $A$ be an elementary abelian group of order $p^{k}$ with $k \geq 3$ acting on a finite $p^{\prime}$-group $G$.

(i) If $\gamma_{k-2}\left(C_{G}(a)\right)$ is nilpotent of class at most $c$ for any $a \in A^{\#}$, then $\gamma_{k-2}(G)$ is nilpotent and has $\{c, k, p\}$-bounded nilpotency class.

(ii) If, for some integer $d$ such that $2^{d}+2 \leq k$, the dth derived group of $C_{G}(a)$ is nilpotent of class at most $c$ for any $a \in A^{\#}$, then the dth derived group $G^{(d)}$ is nilpotent and has $\{c, k, p\}$-bounded nilpotency class.

One indirect evidence in favor of the above conjecture is the result obtained in [6] that the conjecture is true for Lie algebras. Yet, for long time it looked as if the Lie-theoretical result was of no help in dealing with groups. However a breakthrough has occurred with the introduction in [1] of the concept of $A$-special subgroups of a group $G$. In the present paper we combine the use of the subgroups with the Lie-theoretical techniques to show, respectively in Theorems 31 and 41, that both parts of Conjecture 11 are true.

In the next section we mention some standard results on the coprime action of finite groups. We also give the definition of $A$-special subgroups taken from [1] as well as remind the reader some general facts about Lie algebras associated with groups. In Sect. 3 these are used in the proof of Theorem 31. In Sect. 4 we outline the proof of Theorem 41.

\section{Preliminaries}

We start this section with some well-known facts about coprime automorphisms of finite groups. The next two lemmas can be found in [3, 5.3.16, 6.2.2, 6.2.4].

Lemma 21 Let $A$ be a group of automorphisms of the finite group $G$ with $(|A|,|G|)=1$.

1. If $N$ is an A-invariant normal subgroup of $G$, then $C_{G / N}(A)=C_{G}(A) N / N$;

2. If $H$ is an A-invariant $p$-subgroup of $G$, then $H$ is contained in an A-invariant Sylow p-subgroup of $G$.

Lemma 22 Let $p$ be a prime, $G$ a finite $p^{\prime}$-group acted on by an elementary abelian p-group A of rank at least 2. Let $A_{1}, \ldots, A_{s}$ be the maximal subgroups of $A$. If $H$ is an A-invariant subgroup of $G$ we have $H=\left\langle C_{H}\left(A_{1}\right), \ldots, C_{H}\left(A_{s}\right)\right\rangle$. Furthermore if $H$ is nilpotent then $H=\prod_{i} C_{H}\left(A_{i}\right)$.

In [1] we have introduced the concept of an A-special subgroup of a group $G$. The definition is as follows. 
Let $p$ be a prime and $A$ a finite elementary abelian $p$-group acting on a finite group $G$. Let $A_{1}, \ldots, A_{s}$ be the subgroups of index $p$ in $A$ and $H$ a subgroup of $G$. We say that $H$ is an $A$-special subgroup of $G$ of degree 0 if and only if $H=C_{G}\left(A_{j}\right)$ for suitable $j \leq s$. Next, suppose that $i \geq 1$ and the $A$-special subgroups of $G$ of degree $i-1$ are already defined. Then $H$ is an $A$-special subgroup of $G$ of degree $i$ if and only if there exist $A$-special subgroups $J_{1}, J_{2}$ of $G$ of degree $i-1$ such that $H=\left[J_{1}, J_{2}\right] \cap C_{G}\left(A_{j}\right)$ for suitable $j \leq s$.

Here as usual $\left[J_{1}, J_{2}\right]$ denotes the subgroup generated by all commutators $[x, y]$ where $x \in J_{1}$ and $y \in J_{2}$. Of course, the $A$-special subgroups of $G$ are always $A$-invariant. Assume that $A$ has order $p^{k}$. It is clear that for a given integer $i$ the number of $A$-special subgroups of $G$ of degree $i$ is $\{i, k, p\}$-bounded.

Recall some properties satisfied by the $A$-special subgroups of $G$. The proofs can be found in [1].

Proposition 21 Let $A$ be an elementary abelian p-group of order $p^{k}$ with $k \geq 2$ acting on a finite $p^{\prime}$-group $G$ and let $A_{1}, \ldots, A_{s}$ be the maximal subgroups of $A$. Let $i \geq 0$ be an integer.

1. If $i \geq 1$, then every A-special subgroup of $G$ of degree $i$ is contained in some A-special subgroup of $G$ of degree $i-1$.

2. Let $S_{i}$ be the subgroup generated by all A-special subgroups of $G$ of degree $i$. Then $S_{i}=G^{(i)}$.

3. If $2^{i} \leq k-1$ and $H$ is an $A$-special subgroup of $G$ of degree $i$, then $H$ is contained in the ith derived group of $C_{G}(B)$ for some subgroup $B \leq A$ such that $|A / B| \leq p^{2^{i}}$.

In [1] we have also established the following result about generation of an $A$-invariant Sylow subgroup of $G^{(d)}$.

Theorem 21 Let $A$ be an elementary abelian p-group of order $p^{k}$ with $k \geq 2$ acting on a finite $p^{\prime}$-group $G$. Let $r$ be a prime and $R$ an A-invariant Sylow $r$-subgroup of $G^{(d)}$, for some integer $d \geq 0$. Let $R_{1}, \ldots, R_{t}$ be the subgroups of the form $R \cap H$ where $H$ ranges through $A$-special subgroups of $G$ of degree $d$. Then $R=\left\langle R_{1}, \ldots, R_{t}\right\rangle$.

The proofs of the main results of the present paper are based on Lie techniques. Thus, we wish to recall here some useful Lie-theoretic machinery. Throughout the paper the term Lie algebra means Lie algebra over an associative ring with unity. Let $L$ be a Lie algebra and let $X, Y, X_{1}, \ldots, X_{t}$ be subsets of $L$. We denote by $[X, Y]$ the subspace of $L$ spanned by the set $\{[x, y] \mid x \in X, y \in Y\}$ and we write $\left[X_{1}, \ldots, X_{t}\right]$ for $\left[\left[X_{1}, \ldots, X_{t-1}\right], X_{t}\right]$. If $t \geq 2$, we write $\left[X,{ }_{t} Y\right]$ for $\left[\left[X,{ }_{t-1} Y\right], Y\right]$. We denote by $\langle X\rangle$ the subalgebra of $L$ generated by $X$.

Let $G$ be a group and let us denote by $\gamma_{i}$ the $i$ th term of the lower central series of $G$. The associated Lie algebra $L(G)$ of the group $G$ is defined by

$$
L(G)=\bigoplus_{i=1}^{\infty} \gamma_{i} / \gamma_{i+1},
$$

where we write additively the abelian groups $\gamma_{i} / \gamma_{i+1}$. Commutation in the group $G$ induces a well-defined binary operation with respect to which $L(G)$ becomes a Lie ring (Lie algebra over $\mathbb{Z}$ ). The details related to this construction can be found for example in [5]. If the group $G$ is nilpotent, then the Lie algebra $L(G)$ is also nilpotent and has the same nilpotency class as $G$.

Given a subgroup $H$ of $G$, we can associate to $H$ the subalgebra

$$
L(G, H)=\bigoplus_{i=1}^{\infty}\left(H \cap \gamma_{i}\right) \gamma_{i+1} / \gamma_{i+1} \text {. }
$$


If a group $A$ acts on $G$, then $A$ acts naturally also on each quotient $\gamma_{i} / \gamma_{i+1}$ and this action extends uniquely to an action by automorphisms on the whole Lie algebra $L(G)$. Lemma 21(1) shows that if $(|A|,|G|)=1$, then

$$
C_{L(G)}(A)=\bigoplus_{i} C_{\gamma_{i}}(A) \gamma_{i+1} / \gamma_{i+1}
$$

Therefore in the case where $(|A|,|G|)=1$ we have $C_{L(G)}(A)=L\left(G, C_{G}(A)\right)$.

Later on we will require the following lemma.

Lemma 23 Let $L$ be a Lie algebra such that $p L=L$ where $p$ is a prime, and let $A$ be a finite elementary abelian p-group acting by automorphisms on L. Let $A_{1}, \ldots, A_{s}$ be the maximal subgroups of $A$. Suppose that $L$ is generated by $A$-invariant subspaces $R_{1}, \ldots, R_{t}$ with the property that for any integers $i, j$ and $k$ there exists some integer $m$ such that

$$
\left[R_{i}, R_{j}\right] \cap C_{L}\left(A_{k}\right) \leq R_{m} .
$$

Then $L$ is spanned by $R_{1}, \ldots, R_{t}$.

Proof Clearly, $L$ is a linear span of subspaces of the form $\left[R_{i_{1}}, \ldots, R_{i_{w}}\right]$, where $R_{i_{1}}, R_{i_{2}}, \ldots, R_{i_{w}}$ are not necessarily distinct elements of $\left\{R_{1} \ldots, R_{t}\right\}$. So choose $R_{i_{1}}, R_{i_{2}}$, $\ldots, R_{i_{w}} \in\left\{R_{1} \ldots, R_{t}\right\}$ and put $R=\left[R_{i_{1}}, \ldots, R_{i_{w}}\right]$. It is sufficient to show that $R$ is contained in $\sum_{j} R_{j}$. We argue by induction on $w$. If $w=1$, then $R=R_{j}$, for some $j$ and there is nothing to prove. Assume that $w \geq 2$ and put $R_{0}=\left[R_{i_{1}}, \ldots, R_{i_{w-1}}\right]$. Thus $R=\left[R_{0}, R_{i_{w}}\right]$. Since $R$ is an $A$-invariant subspace it follows from Lemma 22 that $R=\sum_{\lambda \leq s} C_{R}\left(A_{\lambda}\right)$. By the inductive hypothesis $R_{0} \leq \sum_{j} R_{j}$. Therefore we have

$$
\begin{aligned}
C_{R}\left(A_{\lambda}\right)=\left[R_{0}, R_{i_{w}}\right] \cap C_{L}\left(A_{\lambda}\right) & \leq\left[\sum_{j} R_{j}, R_{i_{w}}\right] \cap C_{L}\left(A_{\lambda}\right) \\
& \leq \sum_{j}\left(\left[R_{j}, R_{i_{w}}\right] \cap C_{L}\left(A_{\lambda}\right)\right) .
\end{aligned}
$$

By the hypothesis each summand $\left[R_{j}, R_{i_{w}}\right] \cap C_{L}\left(A_{\lambda}\right)$ is contained in $R_{m}$, for some integer $m$, and so it follows that $R \leq \sum_{j} R_{j}$, as desired.

\section{Proof of the main result}

The aim of this section is to prove part (ii) of Conjecture 11 .

Theorem 31 Let $c$ be a positive integer, $p$ a prime, and $A$ an elementary abelian group of order $p^{k}$ with $k \geq 3$ acting on a finite $p^{\prime}$-group $G$. If, for some integer $d$ such that $2^{d}+2 \leq k$, the dth derived group of $C_{G}(a)$ is nilpotent of class at most $c$ for any $a \in A^{\#}$, then the $d$ th derived group $G^{(d)}$ is nilpotent and has $\{c, k, p\}$-bounded nilpotency class.

First we wish to show that under the hypotheses of the above theorem the $d$ th derived group $G^{(d)}$ is nilpotent. In what follows we write $F(K)$ for the Fitting subgroup of a group $K$ and $O_{\pi}(K)$ for the maximal normal $\pi$-subgroup of $K$, where $\pi$ is a set of primes. 
Lemma 31 Assume the hypotheses of Theorem 31. Then $G^{(d)}$ is nilpotent.

Proof Suppose that the lemma is false and let $G$ be a counterexample of minimal order. Since the $d$ th derived group of $C_{G}(a)$ is nilpotent, it follows that $C_{G}(a)$ is soluble for any $a \in A^{\#}$. Therefore Glauberman's result on soluble signalizer functors [2] implies that $G$ is soluble. Assume that $G$ has two distinct minimal $A$-invariant normal subgroups $M_{1}$ and $M_{2}$. By minimality the image of $G^{(d)}$ in $G / M_{1}$ and in $G / M_{2}$ is nilpotent. Thus the image of $G^{(d)}$ must be nilpotent in the quotient $G /\left(M_{1} \cap M_{2}\right)$. This is a contradiction since $M_{1} \cap M_{2}=1$.

Therefore $G$ has a unique minimal $A$-invariant normal subgroup $M$. Again the quotient $G^{(d)} / M$ is nilpotent. It is clear that $M$ is an elementary abelian $q$-group for some prime $q$. Let $A_{1}, \ldots, A_{s}$ be the maximal subgroups of $A$. By Lemma $22 M=M_{1}, M_{2}, \ldots, M_{s}$, where $M_{i}=C_{M}\left(A_{i}\right)$ for $i \leq s$. Since $G^{(d)}$ is not nilpotent, it is not a $q$-group. Therefore by Lemma 21(2) $G^{(d)}$ contains an $A$-invariant Sylow $r$-subgroup $R$ for some prime $r \neq q$. Theorem 21 tells us that $R$ is generated by its intersections with $A$-special subgroups of degree $d$. Thus, $R=\left\langle R_{1}, \ldots, R_{t}\right\rangle$, where $R_{j}=R \cap H_{j}$ for some $A$-special subgroup $H_{j}$ of $G$ of degree $d$. Now fix the integers $i$ and $j$ and consider the subgroup $\left\langle M_{i}, R_{j}\right\rangle$. Since $2^{d} \leq k-1$ it follows from Proposition 21(3) that $H_{j}$ is contained in $C_{G}(B)^{(d)}$ for some subgroup $B$ of $A$ such that $|A / B| \leq p^{2^{d}}$. On the other hand $M_{i} \leq C_{G}\left(A_{i}\right)$ and note that the intersection $B \cap A_{i}$ is not trivial. Therefore there exists $a \in A^{\#}$ such that $M_{i} \leq C_{G}(a)$ and $H_{j} \leq C_{G}(a)^{(d)}$. It follows that $H_{j}$ is contained in $F\left(C_{G}(a)\right)$. Since $M_{i}$ is contained in a normal abelian subgroup of $G$ and also in $C_{G}(a)$, it follows that $\left\langle M_{i}, R_{j}\right\rangle$ is nilpotent. Bearing in mind that $M$ is a $q$-group and $R$ is an $r$-group we deduce that $\left[M_{i}, R_{j}\right]=1$ and this holds for any $i, j$. Recall that $M=M_{1}, M_{2}, \ldots, M_{s}$ and $R=\left\langle R_{1}, \ldots, R_{t}\right\rangle$. Therefore $[M, R]=1$.

The fact that $G^{(d)} / M$ is nilpotent implies that also $C_{G}(M) \cap G^{(d)}$ is nilpotent. Hence, every $q^{\prime}$-element of $C_{G}(M) \cap G^{(d)}$ belongs to $O_{q^{\prime}}(G)$. On the other hand $O_{q^{\prime}}(G)$ is trivial since $M$ is the unique minimal $A$-invariant normal subgroup of $G$. Thus, we obtain a contradiction as we have just shown that $R$ centralizes $M$.

Proof (Proof of Theorem 31) By Lemma $31 G^{(d)}$ is nilpotent. Let $L=L\left(G^{(d)}\right.$ ) be the Lie algebra associated with $G^{(d)}$. Then $p L=L$ and $L$ has the same nilpotency class as $G^{(d)}$. The group $A$ naturally acts by automorphisms on the Lie algebra $L$. From the hypothesis that $C_{G}(a)^{(d)}$ is nilpotent of class at most $c$ we obtain that $C_{L}(a)^{(d)}$ is nilpotent of class at most $c$ for any $a \in A^{\#}$.

Let $K=L \otimes \mathbb{Z}[\omega]$, where $\omega$ is a primitive $p$ th root of unity. Then for each $i \geq 0$ and $a \in A^{\#}$ we have

$$
C_{K}(a)^{(i)}=C_{L}(a)^{(i)} \otimes \mathbb{Z}[\omega] .
$$

Hence, the nilpotency of $C_{L}(a)^{(d)}$ implies that also $C_{K}(a)^{(d)}$ is nilpotent of class at most $c$ for any $a \in A^{\#}$.

We are in the position to apply Theorem 2.7(2) from [6] and conclude that $K^{(d)}$ is nilpotent of $\{c, k, p\}$-bounded class. The same holds for $L^{(d)}$. Let us denote the nilpotency class of $L^{(d)}$ by $e$.

By Proposition 21(2) $G^{(d)}=\left\langle H_{1}, H_{2}, \ldots, H_{t}\right\rangle$, where $H_{i}$ are the $A$-special subgroups of $G$ of degree $d$. Since $2^{d}+2 \leq k$, Proposition 21(3) tells us that each $A$-special subgroup $H_{i}$ of $G$ of degree $d$ is contained in $C_{G}(B)^{(d)}$, for some subgroup $B$ of $A$ such that $|A / B| \leq p^{2^{d}}$. Let $A_{1}, \ldots, A_{s}$ be the maximal subgroups of $A$. For any $A_{j}$ the intersection $B \cap A_{j}$ is not trivial. Thus, there exists $a \in A^{\#}$ such that the centralizer $C_{G}\left(A_{j}\right)$ is contained in $C_{G}(a)$ and 
$H_{i}$ is contained in $C_{G}(a)^{(d)}$. Since $C_{G}(a)^{(d)}$ is nilpotent of class at most $c$ we deduce that

$$
\left[C_{G}\left(A_{j}\right),{ }_{c+1} H_{i}\right]=1 .
$$

Next we define recursively what will be called $A$-subalgebras of $L$. For each $A$-special subgroup $H_{i}$ of $G$ of degree $d$ we consider the corresponding subalgebra $L\left(G^{(d)}, H_{i}\right)$ of $L$ and we define the $A$-subalgebras as follows:

A subalgebra $R$ is an $A$-subalgebra of level 0 if and only if $R=L\left(G^{(d)}, H_{j}\right)$ for suitable $j \leq t$. Next, suppose that $l \geq 1$ and the $A$-subalgebras of level $l-1$ are defined. Then $R$ is an $A$-subalgebra of level $l$ if and only if there exist $A$-subalgebras $R_{1}, R_{2}$ of level $l-1$ such that $R=\left[R_{1}, R_{2}\right] \cap C_{L}\left(A_{j}\right)$ for suitable $j \leq s$. We denote by $\mathcal{R}_{l}$ the set of all $A$-subalgebras of level $l$.

It is clear that every $A$-subalgebra is $A$-invariant and is contained in $C_{L}\left(A_{j}\right)$ for some $j \leq s$. Since $G^{(d)}=\left\langle H_{1}, H_{2}, \ldots, H_{t}\right\rangle$ it follows that $L$ is generated by all $R \in \mathcal{R}_{0}$. It is easy to check that if $R$ is in $\mathcal{R}_{l}$, then $G$ contains an $A$-special subgroup $H$ of degree $d+l$ such that $R \leq L\left(G^{(d)}, H\right)$.

It follows from the definition and Proposition 21(1) that for any $A$-special subgroups $J_{1}$ and $J_{2}$ and for every $j \leq s$ there exists an $A$-special subgroup $J_{3}$ such that

$$
\left[J_{1}, J_{2}\right] \cap C_{G}\left(A_{j}\right) \leq J_{3} .
$$

From this we deduce the corresponding properties of $A$-subalgebras.

(P1) If $l \geq 1$, then every element of $\mathcal{R}_{l}$ is contained in some element of $\mathcal{R}_{l-1}$.

(P2) If $j \leq s$, then for any $R_{1}, R_{2} \in \mathcal{R}_{l}$ there exists $R_{3} \in \mathcal{R}_{l}$ such that

$$
\left[R_{1}, R_{2}\right] \cap C_{L}\left(A_{j}\right) \leq R_{3} .
$$

In the group $G$ we have the relation (1). Therefore in the Lie algebra we have $\left[C_{L}\left(A_{j}\right),{ }_{c+1} L\left(G^{(d)}, H_{i}\right)\right]=0$. Taking into account that every $A$-subalgebra is contained in some $L\left(G^{(d)}, H_{i}\right)$ and that $L=\sum_{j} C_{L}\left(A_{j}\right)$ we deduce $\left[L,{ }_{c+1} L\left(G^{(d)}, H_{i}\right)\right]=0$, and, in particular,

$$
\left[L,{ }_{c+1} R\right]=0
$$

for every $A$-subalgebra $R$.

Let $Q_{l}$ be the subalgebra of $L$ generated by all $R \in \mathcal{R}_{l}$, for $l \geq 0$. The property (P2) and Lemma 23 show that in fact $Q_{l}$ is spanned by all $R \in \mathcal{R}_{l}$. Now we wish to show that $L^{(l)}=Q_{l}$ for any $l \geq 0$. Since $L$ is generated by all $R \in \mathcal{R}_{0}$, this is obvious for $l=0$. Now assume that $l \geq 1$ and use induction on $l$. The inductive hypothesis will be that $L^{(l-1)}=Q_{l-1}$. Let us show that actually $Q_{l}$ is an ideal in $L^{(l-1)}$. Choose $R_{1} \in \mathcal{R}_{l}$ and $R_{2} \in \mathcal{R}_{l-1}$. The properties (P1) and (P2) show that $\left[R_{1}, R_{2}\right] \leq Q_{l}$. Since $L^{(d-1)}=Q_{l-1}$, we conclude that indeed $Q_{l}$ is an ideal in $L^{(l-1)}$. Note that $L^{(l-1)} / Q_{l}$ is abelian. Thus $L^{(l)}=\left[L^{(l-1)}, L^{(l-1)}\right] \leq Q_{l}$. On the other hand, by construction it is clear that $Q_{l}$ is contained in $L^{(l)}$. Hence, $Q_{l}=L^{(l)}$.

We will now prove that $L$ is nilpotent of $\{c, k, p\}$-bounded class. Let $Z=Z\left(L^{(d)}\right)$. Then $[Z, X, Y]=[Z, Y, X]$ for any subsets $X, Y$ of $L^{(d-1)}$. Let $n=\left|\mathcal{R}_{d-1}\right|$, the number of elements in $\mathcal{R}_{d-1}$, and note that the number $n$ is $\{k, p\}$-bounded. Set $r=c n+1$. Since $L^{(d-1)}=\sum_{i \leq n} R_{i}$, we can write

$$
\left[Z,{ }_{r} L^{(d-1)}\right]=\sum\left[Z,{ }_{u_{1}} R_{1}, \ldots, u_{n} R_{n}\right],
$$

where $u_{1}+\cdots+u_{n}=r$ and $R_{1}, \ldots, R_{n}$ are in $\mathcal{R}_{d-1}$. The number $r$ is big enough to ensure that $u_{j} \geq c+1$ for some $j \leq n$. It follows from (3) that each summand in (4) is equal to 
zero. Thus $\left[Z{ }_{r} L^{(d-1)}\right]=0$ and $Z \leq Z_{r}\left(L^{(d-1)}\right)$, where $Z_{r}\left(L^{(d-1)}\right)$ is the $r$ th term of the upper central series of $L^{(d-1)}$. Now repeating this argument for $L^{(d-1)} / Z, L^{(d-1)} / Z_{2}\left(L^{(d)}\right)$ and so on, we conclude that $L^{(d)} \leq Z_{e r}\left(L^{(d-1)}\right)$ and therefore $L^{(d-1)}$ is nilpotent of class at most $e r+1$. After that we repeat the arguments for $L^{(d-2)} / Z\left(L^{(d-1)}\right), L^{(d-2)} / Z_{2}\left(L^{(d-1)}\right)$ etc. After boundedly many repetitions we conclude that $L$ is nilpotent of $\{c, k, p\}$-bounded class.

Finally we remark that since the nilpotency class of $G^{(d)}$ equals that of $L$, the result follows. The proof is complete.

\section{The other part of the conjecture}

In this section we will outline a proof of the following result.

Theorem 41 Let $A$ be an elementary abelian group of order $p^{k}$ with $k \geq 3$ acting on a finite $p^{\prime}$-group $G$. If $\gamma_{k-2}\left(C_{G}(a)\right)$ is nilpotent of class at most $c$ for any $a \in A^{\#}$, then $\gamma_{k-2}(G)$ is nilpotent and has $\{c, k, p\}$-bounded nilpotency class.

The proof of Theorem 41 is very similar to that of Theorem 31. Very often the changes that need to be done are quite obvious and therefore we omit many details. Most essential difference as compared with Theorem 31 is that the role of $A$-special subgroups will now be played by $\gamma$-A-special subgroups of $G$. These were introduced in [1]. Let us recall the definition.

Let $p$ be a prime and $A$ a finite elementary abelian $p$-group acting on a finite group $G$. Let $A_{1}, \ldots, A_{s}$ be the subgroups of index $p$ in $A$ and $H$ a subgroup of $G$. We say that $H$ is a $\gamma$-A-special subgroup of $G$ of degree 1 if and only if $H=C_{G}\left(A_{j}\right)$ for suitable $j \leq s$. Next, suppose that $i \geq 2$ and the $\gamma$-A-special subgroups of $G$ of degree $i-1$ are already defined. Then $H$ is a $\gamma$-A-special subgroup of $G$ of degree $i$ if and only if there exists a $\gamma$-A-special subgroup $J$ of $G$ of degree $i-1$ such that $H=\left[J, C_{G}\left(A_{j}\right)\right] \cap C_{G}\left(A_{n}\right)$ for suitable $j, n \leq s$. Note that for a given integer $i$ the number of $\gamma$-A-special subgroups of $G$ of degree $i$ is $\{i, k, p\}$-bounded. The following properties of $\gamma$-A-special subgroups have been established in [1].

Proposition 41 Let $A$ be an elementary abelian p-group of order $p^{k}$ with $k \geq 2$ acting on a finite $p^{\prime}$-group $G$ and $A_{1}, \ldots, A_{s}$ the maximal subgroups of $A$. Let $i \geq 1$ be an integer.

1. If $i \geq 2$, then every $\gamma$-A-special subgroup of $G$ of degree $i$ is contained in some $\gamma-A$ special subgroup of $G$ of degree $i-1$.

2. Let $S_{i}$ be the subgroup generated by all $\gamma$-A-special subgroups of $G$ of degree $i$. Then $S_{i}=\gamma_{i}(G)$.

3. If $i \leq k-1$ and $H$ is a $\gamma$-A-special subgroup of $G$ of degree $i$, then $H \leq \gamma_{i}\left(C_{G}(B)\right)$ for some subgroup $B \leq A$ such that $|A / B| \leq p^{i}$.

We will also require the following analogue of Lemma 23.

Lemma 41 Let $L$ be a Lie algebra such that $p L=L$ where $p$ is a prime, and let $A$ be a finite elementary abelian p-group acting by automorphisms on L. Let $A_{1}, \ldots, A_{s}$ be the maximal subgroups of $A$. Suppose that $L$ is generated by $A$-invariant subspaces $R_{1}, \ldots, R_{t}$ with the property that for any integers $i, j$ and $k$ there exists some integer $m$ such that

$$
\left[R_{i}, C_{L}\left(A_{j}\right)\right] \cap C_{L}\left(A_{k}\right) \leq R_{m} .
$$

Then $L$ is spanned by $R_{1}, \ldots, R_{t}$. 
Now we sketch out the proof of Theorem 41.

Proof (Proof of Theorem 41) First, we notice that $\gamma_{k-2}(G)$ is nilpotent. The proof of the nilpotency of $\gamma_{k-2}(G)$ is similar to that of Lemma 31. Next, we let $L=L\left(\gamma_{k-2}(G)\right)$ be the Lie algebra associated with $\gamma_{k-2}(G)$. Then $p L=L$ and $L$ has the same nilpotency class as $\gamma_{k-2}(G)$. The group $A$ naturally acts by automorphisms on $L$ and, since $\gamma_{k-2}\left(C_{G}(a)\right)$ is nilpotent of class at most $c$, it follows that $\gamma_{k-2}\left(C_{L}(a)\right)$ is nilpotent of class at most $c$ for any $a \in A^{\#}$. Put $K=L \otimes \mathbb{Z}[\omega]$, where $\omega$ is a primitive $p$ th root of unity. The nilpotency of $\gamma_{k-2}\left(C_{L}(a)\right)$ implies that also $\gamma_{k-2}\left(C_{K}(a)\right)$ is nilpotent of class at most $c$ for any $a \in A^{\#}$. Theorem 2.7(1) of [6] now tells us that $\gamma_{k-2}(K)$ is nilpotent of $\{c, k, p\}$-bounded class. Hence, also the nilpotency class of $\gamma_{k-2}(L)$ is $\{c, k, p\}$-bounded. We denote the nilpotency class of $\gamma_{k-2}(L)$ by $e$.

Let $H_{1}, H_{2}, \ldots, H_{t}$ be the $\gamma$-A-special subgroups of $G$ of degree $k-2$. By Proposition 41(2) $\gamma_{k-2}(G)=\left\langle H_{1}, H_{2}, \ldots, H_{t}\right\rangle$. Since $k-2 \leq k-1$, Proposition 41(3) tells us that each subgroup $H_{i}$ is contained in $\gamma_{k-2}\left(C_{G}(B)\right)$ for some subgroup $B$ of $A$ such that $|A / B| \leq p^{k-2}$. Let $A_{1}, \ldots, A_{s}$ be the maximal subgroups of $A$. For any $A_{j}$ the intersection $B \cap A_{j}$ is not trivial. Thus, there exists $a \in A^{\#}$ such that the centralizer $C_{G}\left(A_{j}\right)$ is contained in $C_{G}(a)$ and $H_{i}$ is contained in $\gamma_{k-2}\left(C_{G}(a)\right)$. Since $\gamma_{k-2}\left(C_{G}(a)\right)$ is nilpotent of class at most $c$, we have

$$
\left[C_{G}\left(A_{j}\right),{ }_{c+1} H_{i}\right]=1 .
$$

Next, we define recursively what will be called $\gamma$-A-subalgebras of $L$. The definition is similar to that of $A$-subalgebras used in the previous section.

For each $\gamma$-A-special subgroup $H_{i}$ of $G$ of degree $k-2$ we consider the corresponding subalgebra $L\left(\gamma_{k-2}(G), H_{i}\right)$. A subalgebra $R$ is a $\gamma$-A-subalgebra of level 1 if and only if there exists $j \leq t$ such that $R=L\left(\gamma_{k-2}(G), H_{j}\right)$. Further, suppose that $l \geq 2$ and the $\gamma-A$ subalgebras of level $l-1$ are already defined. Then $R$ is a $\gamma-A$-subalgebra of level $l$ if and only if there exists a $\gamma$-A-subalgebra $R_{1}$ of level $l-1$ such that $R=\left[R_{1}, C_{L}\left(A_{j}\right)\right] \cap C_{L}\left(A_{m}\right)$ for suitable $j, m \leq s$.

Since (5) holds in the group $G$, we deduce that $\left[L,{ }_{c+1} R\right]=0$ for every $\gamma$-A-subalgebra $R$. Furthermore, using Lemma 41 one can show that for every $l \geq 1$ the $l$ th term $\gamma_{l}(L)$ of the lower central series of $L$ is spanned by the $\gamma$-A-subalgebras of level $l$.

Finally, we use the above remarks to prove that $L$ is nilpotent of $\{c, k, p\}$-bounded class. This part of the proof is pretty much the same as that of Theorem 31. Since $\gamma_{k-2}(G)$ has the same nilpotency class as $L$, the theorem follows.

\section{References}

1. Acciarri, C., Shumyatsky, P.: Fixed points of coprime operator groups. J. Algebra 342, 161-174 (2011)

2. Glauberman, G.: On solvable signalizer functors in finite groups. Proc. Lond. Math. Soc. 33, 1-27 (1976)

3. Gorenstein, D.: Finite Groups. Chelsea Publishing Company, New York (1980)

4. Guralnick, R., Shumyatsky, P.: Derived subgroups of fixed points. Israel J. Math. 126, 345-362 (2001)

5. Khukhro, E.I.: Nilpotent Groups and Their Automorphisms. de Gruyter-Verlag, Berlin (1993)

6. Shumyatsky, P.: Finite groups and the fixed points of coprime automorphisms. Proc. Am. Math. Soc. 129(12), 3479-3484 (2001)

7. Ward, J.N.: On finite groups admitting automorphisms with nilpotent fixed-point group. Bull. Aust. Math. Soc. 5, 281-282 (1971)

8. Ward, J.N.: On finite soluble groups and the fixed-point groups of automorphisms. Bull. Aust. Math. Soc. 5, 375-378 (1971) 